\title{
Aperture Synthesis Radar Imaging for Upper Atmospheric Research
}

\author{
D. L. Hysell and J. L. Chau \\ Earth and Atmospheric Sciences, Cornell University, Ithaca, New York \\ Jicamarca Radio Observatory, Lima \\ U.S.A., Peru
}

\section{Introduction}

Radars used for upper-atmospheric applications can be engineered to measure the Doppler spectra of their targets adequately for most intents and purposes, the spectral resolution being limited only by the observing time and the constraints of stationarity. Likewise, they can measure the range to their targets adequately for most intents and purposes, range resolution being limited by system bandwidth, the power budget, and the constraints of stationarity. Problems arise for "overspread" targets, where range and frequency aliasing cannot simultaneously be avoided using pulse-to-pulse methodologies, and more complicated pulse-to-lag or aperiodic pulsing methods are required (see for example (Farley, 1972; Huuskonen et al., 1996; Lehtinen, 1986; Sulzer, 1986; Uppala, 1993)). Important examples of this situation include incoherent scatter experiments (Farley, 1969), observations of meteor head echoes (Chau \& Woodman, 2004), and observations of plasma density irregularities present in certain rapid flows, as are found in the equatorial ionosphere during so-called "equatorial spread F" (Woodman, 2009; Woodman \& La Hoz, 1976).

Where capabilities are most limited is in bearing determination and the associated problems of imaging in the directions transverse to the radar beam. Electronic beam steering using phased-array radars offers a means of radar imaging (e.g. Semeter et al. (2009)), but the number of pointing positions that can be used is limited by the incoherent integration time required for each position. If the power budget permits, transmission can be done using a broad beam, and beam forming can be done "after the fact", such that all pointing positions are examined simultaneously (e.g. Kudeki \& Woodman (1990)). Even so, the angular resolution will be limited by the size of the antenna array unless the diffraction limit is removed through numerical deconvolution. The half-power beamwidth of large-aperture radars used for upper atmospheric research is usually of the order of one degree. At ionospheric altitudes, this translates to a transverse resolution of a few to a few tens of kilometers, which may be larger than the scales at which primary plasma waves are excited. The resolution of medium-sized and small research radars with their relatively smaller antenna arrays is relatively poorer still. In applications involving coherent scatter from plasma density irregularities, targets of interest may exhibit backscatter intensities spanning $30 \mathrm{~dB}$ or more of dynamic range. For such targets, the $3 \mathrm{~dB}$ beamwidth of the antenna is essentially irrelevant, and even targets in the sidelobes of the antenna radiation pattern can contribute to the power assigned to a given 
pointing direction. This poses challenges for observing plasma irregularities with important scale sizes of a kilometer or less, which is often the case in ionospheric research.

Aperture synthesis radar imaging utilizes spaced-antenna data to construct true images of the scatterers versus bearing. Some approaches are adaptive, and some achieve "super resolution" by incorporating the effects of diffraction in the analysis. All information about range and Doppler shift can be retained, meaning that the images can be four dimensional, not counting the time axis. As the techniques can synthesize large apertures from small, sparsely-distributed sensors, they may be especially beneficial for small and medium-sized radars, although some of the benefits can only be realized when high signal-to-noise ratios are available.

In this paper, we review the formulation of the radar imaging problem, which is based on concepts and language derived from radar interferometry and radio astronomy. As radar imaging belongs to the class of problems known as inverse problems, some of the ideas from that domain are also reviewed. The factors that govern the resolution achievable in practice will be described, and optimal strategies for sensor placement will be discussed. Error analysis in radar imaging is treated, and some extensions to the basic imaging procedure are outlined. Finally, examples of radar imaging implementations are drawn from upper atmospheric and ionospheric applications. Application in the lower atmosphere exist as well but will not be covered here (Chau \& Woodman, 2001; Hassenpflug et al., 2008; Palmer et al., 1998).

\section{Imaging problem}

The imaging problem has been formulated by (Thompson, 1986), and we follow his treatment below. We consider the far-field problem only and regard the backscatter in a given range gate as a random process constituted by plane waves with sources that are statistically uncorrelated and distributed in space. Imaging data have the form of complex interferometric cross spectra obtained from spaced antenna pairs separated by a vector distance d. Such "visibility" measurements $V\left(k \mathbf{d}, f_{D}\right)$ are related to the "brightness" distribution $B\left(\hat{\sigma}, f_{D}\right)$, the scattered power density as a function of bearing and Doppler frequency, by

$$
V\left(k \mathbf{d}, f_{D}\right)=\int A_{N}(\hat{\sigma}) B\left(\hat{\sigma}, f_{D}\right) e^{j k \mathbf{d} \cdot \hat{\sigma}} d \Omega
$$

where $k$ is the wavenumber, $f_{D}$ is the Doppler frequency, and $\hat{\sigma}$ is a unit vector in the direction of the bearing of interest and where the integration is over all solid angles in the upper half space. As different Doppler spectral components of the data are treated independently, we omit $f_{D}$ in the formalism that follows.

In (1), $A_{N}$ is the normalized two-way antenna effective area. In radar imaging, the antennas used for reception are typically much smaller than the antennas used for transmission, and $A_{N}$ is consequently dominated by the characteristics of the transmitting antenna array. Together, the product $A_{N} B$ is the effective brightness distribution, $B_{\text {eff, which represents the angular }}$ distribution of the received signals. It is this quantity that are interested in recovering from the data, the antenna radiation patterns being known. The radiation pattern need only be treated explicitly when heterogeneous receiving antennas are used (see below). 
Equation (1) resembles a Fourier transform between baseline and bearing space. In Cartesian coordinates, (1) is

$$
\begin{aligned}
& V\left(k d_{x}, k d_{y}, k d_{z}\right) \\
& =\int \frac{B_{\text {eff }}(\eta, \xi)}{\sqrt{1-\eta^{2}-\xi^{2}}} e^{j k\left(d_{x} \eta+d_{y} \xi+d_{z} \sqrt{1-\eta^{2}-\xi^{2}}\right)} d \eta d \xi
\end{aligned}
$$

where $\eta$ and $\xi$ are the direction cosines of $\hat{\sigma}$ with respect to the $x$ and $y$ coordinates, which can be oriented arbitrarily. If the field of view of the sky being considered is sufficiently restricted, the radical in the denominator of the integrand can be regarded as a constant. Then, if the spaced receivers are coplanar so that all $d_{z}$ can be made to be zero, or if the brightess is finite only where $\eta$ and $\psi$ are small, (2) becomes a two-dimensional Fourier transform. This condition is required for "fringe stopping," the practice of calibrating the complex gains of the sensor channels so as to remove the fringes in the visibility spectrum of a point calibration target (see phase calibration section below). If $B_{\text {eff }}$ is limited by the finite width of the radar radiation pattern, the visibilities can be completely represented by a discrete set of periodic visibility measurements.

\section{Inverse methods}

Equation (2) shows that the radar imaging problem is actually the linear problem of inverting a Fourier transform. Since visibility data are generally acquired sparsely and incompletely, inversion of (2) by means of a discrete Fourier transform algorithm is generally impossible and would be undesirable in any case unless that algorithm were followed by another one to deconvolve the radar radiation pattern from the resulting image. A number of somewhat ad-hoc approaches to Fourier analysis of sparsely sampled data have emerged, including the Lomb periodogram (Lomb, 1976) and CLEAN (Högbom, 1974), which amount to linear least-squares fitting of representer functions to the data. Performance of such algorithms is uneven and suffers from pathologies inherent in inverse problems that have not been accounted for. The pathologies arise in part from the fact that the measured visibilities contain noise that is not incorporated in (2), which is therefore only an approximation.

As (2) is a linear transformation, the discrete visibility data could be mapped to a discretized version of the brightness distribution through a matrix $(G)$ whose properties would describe the characteristics of the inverse solution. For example, if the column space of $G$ is incomplete, there may be no model brightness that can reproduce the measured visibilities, the problem would be over-determined, and no solution would exist. If the row space of $G$ is incomplete, then it would be possible to add features to the brightness without altering the predicted visibilities, the problem would be under-determined, and solutions to the inverse problem would not be unique.

More generally, both the column and row spaces of $G$ may be incomplete, meaning that the problem is simultaneously over- and under-determined (mixed determined or rank deficient) and that any number of candidate brightness distributions might give acceptably close approximations to the desired solution in terms of a chi-squared prediction error metric. If $G$ is also poorly conditioned, then those candidates could vary widely, and the inversion would be unstable.

Inverse methods are required for mixed-determined, poorly-conditioned problems (see e.g. Aster et al. (2005); Menke (1984); Tarantola (1987) for a review). The strategy generally 
amounts to reducing the candidate space of model solutions by imposing a priori information. The information may involve expectations about the variance of the model solution (its "roughness" or regularity) or something more specific, such as the range of admissible numerical values it can assume. A priori information may be incorporated implicitly through the inclusion of damping terms in the inversion algorithm (e.g. damped least squares, Tikhonov regularization, etc.) or explicitly using a Bayesian formalism. Other desirable properties, including model-data consistency, model resolution, and data resolution, can also be optimized.

A common approach to radar imaging is based on the linear constrained minimum variance (LCMV) (or sometimes minimum variance distortionless response (MVDR)) principle and was introduced by Capon (1969). Consider the column vector $\mathbf{x}$ with $n$ entries corresponding to complex voltage samples from $n$ sensors, each at a coordinate $\mathbf{r}_{n}$ measured from some reference point. Suppose the objective is to discriminate echoes arriving along a wavevector $\mathbf{k}$ from other echoes, noise, interference, etc., by forming an appropriated weighted sum of the voltage samples, $y=w^{\dagger} x$, prior to detection. If the weights are all unity, the signals detected by the sensors from a point source designated by $\mathbf{k}$ would be proportional to the column vector with elements $e^{i \mathbf{k} \cdot \mathbf{r}_{1}}, e^{i \mathbf{k} \cdot \mathbf{r}_{2}}, \cdots, e^{i \mathbf{k} \cdot \mathbf{r}_{n}}$. After incoherent integration, the output of the detector with arbitrary weights will be

$$
\left\langle\left|y^{2}\right|\right\rangle=w^{\dagger}\left\langle x x^{\dagger}\right\rangle w=w^{\dagger} R w
$$

where $w$ is the column vector composed of the weights, $R$ is the signal covariance matrix, constructed from the measured visibilities, and + denotes the complex conjugate transpose.

Capon's LCMV strategy is to optimize the weights by minimizing the output of the detector while maintaining unity gain in the direction of the point source, viz.

$$
\mathbf{w}=\underset{\mathbf{w}, \gamma}{\arg \min }: w^{\dagger} R w+\gamma\left(w^{\dagger} e-1\right)
$$

where $\gamma$ is a Lagrange multiplier. The unity-gain constraint is imposed to prevent the trivial solution. The output of the optimal detector using the weights thus found for a given bearing can readily be shown to be $\left\langle|y|^{2}\right\rangle=\left(e^{\dagger} R^{-1} e\right)^{-1}$. Imaging then is performed by computing the optimized detector output for all possible bearings. The algorithm is essentially a linear beam former, where nulls are adaptively aligned with sources that are not aligned with the bearing of interest.

Capon's LCMV method is simple to implement and execute computationally. While there is no provision for error handling in the algorithm posed above, the remedy is to precompute the visibility error covariance matrix (see below) and then transform (3) through similarity transformation into a space where that matrix is the identity. The method is equally well suited for imaging continua and point targets, making it a superior choice for geophysical remote sensing compared to point-targeting algorithms like MUSIC (Schmidt, 1986) or CLEAN. However, there is no guarantee that the brightness distribution found will be consistent with the visibility data within the tolerance of the specified error bounds. The a priori information contained within the method is moreover far from explicit, making it hard to assess its validity.

Any number of alternative imaging methods exist that can minimize or constrain the model prediction error while managing issues arising from the mixed determined or ill conditioned nature of the inverse problem. In the next section of the paper, we turn our attention to the 
MaxEnt algorithm, which does not suffer from the limitations of Capon's method and which possesses a number of other desirable features as a consequence of the incorporation of rather informative prior information.

\section{MaxEnt formulation}

The algorithm described below derives from the MaxEnt spectral analysis method, a Bayesian method based on maximizing the Shannon entropy of the spectrum (Shannon \& Weaver, 1949). The method should not be confused with the maximum entropy method (MEM or ME) or similar autoregressive models, with which it has only a remote connection (Jaynes, 1982). MaxEnt was originally applied to spaced-receiver imaging by Gull \& Daniell (1978) and also by Wernecke \& D'Addario (1977) in a more generalized way. Variations on the technique were published later by Wu (1984), Skilling \& Bryan (1984), and Cornwell \& Evans (1985). MaxEnt is now applied to a wide array of problems, including natural language processing (NLP), quantum physics, and climate science, to name a few.

Expansive rationales for MaxEnt have been given by Ables (1974), Jaynes (1982; 1985), Skilling (1991), and Daniell (1991), among others. MaxEnt is a Bayesian optimization technique that maximizes the MAP (maximum a posteriori) probability of an image given prior probability rooted in Shannon's entropy and constraints related to the model prediction error, error bounds, image support, certain normalization, and other factors. As entropy admits only globally positive brightness distributions, it rejects the vast majority of candidate solutions in favor of a small, allowable solution subspace. The entropy metric favors uniform images in the absence of contrary information but is nevertheless edge preserving. Moreover, the use of entropy for prior probability makes the algorithm minimally dependent on unknown quantities and, in that sense, bias and artifact free. It is a formalization of Occam's razor.

The algorithm described here is based on one developed by Wilczek \& Drapatz (1985) (WD85) for radio astronomy. The real valued brightness will be represented by the symbol $f_{i}=f\left(\theta_{i}\right)$. The visibility data come from normalized cross-correlation estimates

$$
V\left(k \mathbf{d}_{j}\right)=\frac{\left\langle v_{1} v_{2}^{*}\right\rangle}{\sqrt{\left\langle\left|v_{1}\right|^{2}\right\rangle-N_{1}} \sqrt{\left\langle\left|v_{2}\right|^{2}\right\rangle-N_{2}}}
$$

where the $v_{1,2}$ represent quadrature voltage samples from a pair of receivers spaced by a distance $\mathbf{d}_{j}$ and $N_{1,2}$ are the corresponding noise estimates. The angle brackets above are the expectation. We will represent the visibility data by the symbol $g_{j}=g\left(k \mathbf{d}_{j}\right)$ and assign two real values for each baseline; one each for the real and imaginary part of (5). Given $M$ interferometry baselines with nonzero length, there will be a total of $2 M+1$ distinct visibility data. (The visibility for the zero baseline is identically unity.)

In matrix notation, (2) may be expressed as

$$
g^{t}+e^{t}=f^{t} h
$$

where $g, e$, and $f$ are column vectors and $t$ represents the transpose. Here, the elements of the matrix $h\left(h_{i j}\right)$ are the real or the imaginary part of the point spread function $\exp \left(i k \mathbf{d}_{j}\right.$. $\left.\hat{\sigma}_{i}\right)$, depending on whether $g_{j}$ is the real or imaginary part of (5), and $e_{j}$ represents the corresponding random error arising from the finite number of samples used to estimate (5). The elements $f_{i}$ of vector $f$ represent the brightness distribution evaluated across the defined image space. 
MaxEnt explicitly associates the prior probability of a candidate image with the Shannon entropy of the brightness distribution, $S=-\sum_{i} f_{i} \ln \left(f_{i} / F\right)$. Here, $F=f^{t} 1=g_{0}=g(0)$ is the total image brightness (" 1 " being a column with unity elements). Of all distributions, the uniform one has the highest entropy. In that sense, entropy is a smoothness metric. The entropy of an image is also related to the likelihood of occurrence in a random assembly process. All things being equal, a high-entropy distribution should be favored over a low entropy one. The former represents a broadly accessible class of solutions, while the latter represents an unlikely outcome that should only be considered if the data demand it. Finally, only non-negative brightness distributions are allowed by $S$. In incorporating it, we reject the vast majority of candidate images in favor of a small subclass of physically obtainable ones.

Neglecting error bounds for the moment, the brightness distribution that maximizes $S$ while being constrained by (6) is the extremum of the functional:

$$
E(f(\lambda, L))=S+\left(g^{t}-f^{t} h\right) \lambda+L\left(f^{t} 1-F\right)
$$

where the $\lambda$ is a column vector of Lagrange multipliers introduced to enforce the constraints by the principles of variational mechanics and $L$ is another Lagrange multiplier enforcing the normalization of the brightness. Maximizing (7) with respect to the $f_{i}$ and to $L$ yields a model for the brightness, parametrized by the $\lambda_{j}$ :

$$
\begin{aligned}
& f_{i}=F \frac{e^{-[h \lambda]_{i}}}{Z} \\
& Z=\sum_{i} e^{-[h \lambda]_{i}}
\end{aligned}
$$

where we note how $Z$ plays the role of Gibbs' partition function here.

Statistical errors are accounted for in WD85 by adapting (7) to enforce a constraint on the expectation of $\chi^{2}$. The constraint is incorporated with the addition of another Lagrange multiplier $(\Lambda)$. The constraint regarding the normalization of the brightness is enforced by the form of $f$ resulting from (8) and need not be enforced further.

$$
\begin{aligned}
& E(f(e, \lambda, \Lambda)) \\
& =S+\left(g^{t}+e^{t}-f^{t} h\right) \lambda+\Lambda\left(e^{t} C e-\Sigma\right) \\
& =\left(g^{t}+e^{t}\right) \lambda+F \ln Z+\Lambda\left(e^{t} C e-\Sigma\right)
\end{aligned}
$$

where the last step was accomplished by substituting (8) and (9) into $S$. The $\Sigma$ term constrains the error norm, calculated in terms of theoretical error covariance matrix $C$, which we take to be diagonal. Rather than finding the brightness with the smallest model prediction error which also has a high entropy, WD85 finds the brightness which deviates from the data in a prescribed way so as to have the highest possible entropy consistent with experimental uncertainties.

Maximizing (10) with respect to the Lagrange multipliers yields $2 M+1$ algebraic equations:

$$
g^{t}+e^{t}-f^{t} h=0
$$

which merely restates (6). Maximizing with respect to the error terms in $e$ yields equations relating them to the elements of $\lambda$ :

$$
\lambda+2 \Lambda C^{-1} e=0
$$


(no sum implied). Maximizing with respect to $\Lambda$ yields one more equation relating that term to the others.

$$
4 \Sigma \Lambda^{2}-\lambda^{t} C \lambda=0
$$

The resulting system of $2 M+1$ coupled, nonlinear equations for the Lagrange multipliers can be solved numerically. (The algorithm implemented here uses the hybrid method of Powell (1970).) Finally, (8) yields the desired image. The algorithm is robust and converges in practice when provided with data uncontaminated by interference. An analytic form of the required Jacobian matrix can readily be derived from (11).

\subsection{Error analysis}

Defining $\rho_{12}$ as the normalized cross-correlation of the signals from receivers 1 and 2, an obvious estimator of $\rho_{12}$ is:

$$
\hat{\rho}_{12}=\frac{\frac{1}{m} \sum_{i=1}^{m} v_{1 i} v_{2 i}^{*}}{\sqrt{\frac{1}{m} \sum_{i=1}^{m}\left|v_{1 i}\right|^{2} \frac{1}{m} \sum_{i=1}^{m}\left|v_{2 i}\right|^{2}}}
$$

where the numerator and denominator are computed from the same $m$ statistically independent, concurrent samples. The error covariance matrix for interferometric cross-correlation or cross-spectral visibility estimates derived from this estimator was given by Hysell \& Chau (2006):

$$
\begin{aligned}
& \left\langle e_{r 12} e_{r 34}\right\rangle=\Re\left(\delta^{2}+\delta^{\prime 2}\right) / 2 \\
& \left\langle e_{i 12} e_{i 34}\right\rangle=\Re\left(\delta^{2}-\delta^{\prime 2}\right) / 2 \\
& \left\langle e_{r 34} e_{i 12}\right\rangle=\Im\left(\delta^{2}+\delta^{\prime 2}\right) / 2 \\
& \left\langle e_{r 12} e_{i 34}\right\rangle=\Im\left(\delta^{\prime 2}-\delta^{2}\right) / 2
\end{aligned}
$$

where $e_{r 12}$ stands for the error in the estimate of the real part of the correlation of the signals from spaced receivers 1 and 2, for example, and where the indices may be repeated depending on the interferometry baselines in question. Also,

$$
\begin{aligned}
\delta^{2}= & \frac{1}{m}\left[\rho_{13} \rho_{24}^{*}-\frac{1}{2} \rho_{34}^{*}\left(\rho_{13} \rho_{23}^{*}+\rho_{14} \rho_{24}^{*}\right)\right. \\
& -\frac{1}{2} \rho_{12}\left(\rho_{13} \rho_{14}^{*}+\rho_{23} \rho_{24}^{*}\right) \\
& \left.+\frac{1}{4} \rho_{12} \rho_{34}^{*}\left(\left|\rho_{13}\right|^{2}+\left|\rho_{14}\right|^{2}+\left|\rho_{23}\right|^{2}+\left|\rho_{24}\right|^{2}\right)\right]
\end{aligned}
$$

and

$$
\begin{aligned}
\delta^{\prime 2}= & \frac{1}{m}\left[\rho_{14} \rho_{23}^{*}-\frac{1}{2} \rho_{34}\left(\rho_{13} \rho_{23}^{*}+\rho_{14} \rho_{24}^{*}\right)\right. \\
& -\frac{1}{2} \rho_{12}\left(\rho_{13}^{*} \rho_{14}+\rho_{23}^{*} \rho_{24}\right) \\
& \left.+\frac{1}{4} \rho_{12} \rho_{34}\left(\left|\rho_{13}\right|^{2}+\left|\rho_{14}\right|^{2}+\left|\rho_{23}\right|^{2}+\left|\rho_{24}\right|^{2}\right)\right],
\end{aligned}
$$


$\rho_{12}$ representing the complex correlation of the signals from spaced receivers 1 and 2 , for example. In practice, these terms must be based on experimental estimates. The overall stability of error estimators based on data with statistical errors themselves has not been considered.

\subsubsection{Added noise}

The formulas above were derived in the absence of system noise but can easily be generalized to include noise. The normalized correlation function error covariances for signals in the presence of noise are still given by (19) and (20), only substituting the factor

$$
\rho_{S i i} \rightarrow \frac{S+N}{S}
$$

wherever correlation terms with repeated indices appear. Here, $S$ and $N$ refer to the signal and noise power, respectively.

On the whole, this analysis shows that the error covariance matrix is diagonally dominant only in cases where either the signal-to-noise ratio or the coherence is small. These limits are seldom applicable to coherent scatter, however. Even the longest interferometry baseline at Jicamarca, nearly 100 wavelengths long, very often exhibits high coherence, and even small, portable coherent scatter radars typically run in the high SNR limit. Since the error covariance is not diagonally dominant in general, neglecting off-diagonal terms misrepresents statistical confidence and could lead to image distortion.

In practice, it is expedient to diagonalize the error covariance matrix computed using the formulas above and to apply the corresponding similarity transformation to forward problem stated in (6) (Hysell \& Chau, 2006). We find that the error variances that result fall into two groups with relatively smaller and larger values, respectively. The former correspond roughly to errors associated with measuring interferometric coherence, and the latter to errors associated with interferometric phase.

\subsubsection{Error propagation}

Error propagation through MaxEnt can be treated as follows (see for example Hysell (2007); Silver et al. (1990)). Using Bayes' theorem, we can cast the MaxEnt optimization problem posed in (10) as one of maximizing the posterior probability of a model image, $m$, based on visibility data $d$, which are related linearly through $d=G m$, in the form

$$
\begin{aligned}
p(m \mid d) & \propto e^{S / \Gamma} e^{-\frac{1}{2} e^{t} C^{-1} e} \\
& \equiv e^{-E}
\end{aligned}
$$

where the entropy $S$ is the prior probability and the chi-squared model prediction error is transitional probability. The constant $\Gamma$ weights the two probabilities and must be adjusted according to some criteria. In the variational approach to the optimization problem outlined above, the Lagrange multiplier $\Lambda$ plays the role of $\Gamma$. That variable is controlled by $\Sigma$, and so there is always an adjustable free parameter.

Consider small departures $\delta m$ about the maximum probability (minimum $E$ ) solution. In the neighborhood of a maximum, the gradient of the argument $E$ vanishes, and we can always 
expand

$$
\begin{aligned}
p(m \mid d) & \propto e^{-\frac{1}{2} \delta m^{t} H \delta m} \\
H & =\frac{\partial^{2} E(m)}{\partial \delta m \partial \delta m}
\end{aligned}
$$

with $H$ the Hessian matrix. Now, (24) has the form of a probability density function (PDF) for normally distributed model errors $\delta m$ which we maximize through the minimization of $E$. We can consequently identify the Hessian matrix with the inverse model covariance matrix $C_{m}^{-1}$. Taking the necessary derivatives gives the error bounds on the image:

$$
C_{m}^{-1}=G^{t} C^{-1} G+[\Gamma I m]^{-1}
$$

The $\Gamma$ term in (12) represents the influence of the data on the final MaxEnt model. The greater the influence, the smaller the uncertainties. The other term comes from the entropy prior and ensures that the model variances will be very small where the model values themselves are small. This is obviously significant in view of the importance of suppressing spurious artifacts.

\subsection{Extensions}

Hysell \& Chau (2006) introduced two improvements to WD85 important for upper atmospheric radar research. The first involves incorporating the overall two-way antenna radiation pattern in the imaging analysis. Rather than attempting to remove the two-way pattern from the effective brightness distribution through division, with the attendant conditioning problems, we just acknowledge the influence of the pattern on the effective brightness and modify the entropy metric accordingly to anticipate it. If Shannon's expression favors a uniform brightness distribution, the expression that favors distributions that resemble the beam shape is (Skilling, 1989)

$$
S^{\prime}=-\sum_{i} f_{i} \ln \left(f_{i} / p_{i} F\right)
$$

where $p_{i}$ represents the two-way radiation power pattern. Propagating this expression through the preceding analysis alters only the brightness model:

$$
\begin{aligned}
& f_{i}=F p_{i} \frac{e^{-[h \lambda]_{i}}}{Z} \\
& Z=\sum_{i} p_{i} e^{-[h \lambda]_{i}}
\end{aligned}
$$

The remaining formalism is unchanged. The only restriction is that $p_{i}$ should be positive. In practice, the effect of the modification is to suppress spurious brightness outside in regions where the radiation pattern is depressed.

The second improvement applies when heterogeneous antennas are used for reception. In that case, the $p_{i}$ in (26) can be made to match the radiation pattern of the transmitting antenna array, which is common to all the received signals. The radiation patterns of the receiving antennas are then explicitly incorporated into the expressions for the effective brightness, $B_{\text {eff, }}$ associated with each baseline. In view of (1) or (6), this is done by modifying the point 
spread function for the given baseline $j$ such that $h_{i j} \rightarrow h_{i j} \wp_{1 i} \wp_{2 i}$, where $\wp_{1,2 i}$ are the radiation amplitude patterns for the antennas at either end of the baseline. Given the principle of pattern multiplication, characteristics of the radiation pattern common to all the receiving antennas can equally well be incorporated in $p_{i}$ instead of $\wp_{1,2 i}$.

\subsection{Super-resolution}

That radar imaging resolution does not need to be diffraction limited can be appreciated by considering coherence (normalized visibility) measurements made with a single interferometry baseline in the high signal-to-noise ratio, high coherence limit. As shown by Farley \& Hysell (1996), the mean-squared error for the coherence estimate in this limit is

$$
\delta^{2}=\frac{1}{m}\left[\frac{N}{S}+\frac{1}{2} \epsilon+\mathcal{O}\left(\epsilon^{2}, \frac{N^{2}}{S^{2}}, \epsilon \frac{N}{S}, \cdots\right)\right]
$$

where $\epsilon \equiv 1-|V|^{2}, m$ is the number of statistically independent samples used, and where $S$ and $N$ are the signal and noise powers, respectively. Even given a finite number of samples, the coherence estimate for a highly coherent target can be arbitrarily accurate given a high enough signal-to-noise ratio. This means that the angular width of narrow targets can be measured arbitrarily well, regardless of the baseline spacing, if $S / N$ is sufficiently high. Insofar as imaging, an inverse method that accounts for the effects of diffraction in the forward model (i.e. the point spread function) need not be diffraction limited.

On the basis of information theory pertaining to the rate of information transmission through a noisy channel, Kosarev (1990) investigated the resolution limit for spectral analysis, deriving Shannon's resolution limit:

$$
S R=\frac{1}{3} \log _{2}(1+S / N)
$$

This metric represents the maximum achievable resolution improvement over the diffraction-limited, noise-free case for non-parametric signal processing methods. Kosarev (1990) argued that there is no contradiction between this limit and the Heisenberg uncertainty principle. Kosarev (1990) furthermore performed numerical tests, comparing a spectral recovery algorithm based on maximum likelihood with entropy prior probability. Over the $S / N$ range from 10-50 dB, the algorithm was able to achieve the Shannon limit. At Jicamarca, the longest interferometry baseline is nearly 100 wavelengths long, and the diffraction limited resolution is consequently about $0.5^{\circ}$. In practice, useful resolution at about the $0.1^{\circ}$-level can be obtained with strong backscatter.

\subsection{Optimal sensor placement}

The placement of sensors (receiving antennas or antenna groups) on the ground is typically constrained by practical consideration. If the sensors are subarrays of a fixed phased array, as in the case of the Jicamarca Radio Observatory in Peru or the MU Radar in Japan, a number of modules set by the number of receivers available will be selected from the total available in such a way as to avoid redundant baselines. To avoid ambiguity, baseline lengths can be selected such that the interferometry sidelobes are not illuminated by the transmitting antenna. Baseline orientations may be selected to accommodate anisotropies in the scatterers. As a rule, uniform sampling of visibility space seems to be conducive to artifact reduction, although there may be good reasons to deviate from it. 
Sharif \& Kamalabadi (2008) studies the optimal placement of sensors for different remote sensing applications, including aperture synthesis imaging. They considered data inversion through Tikhonov regularization, which is similar to the methodology discussed here except with Gaussian model statistics replacing model entropy as the prior probability. This permitted an entirely linear formulation of the problem. Included in the imaging problem were allowances for constraints on image smoothness and support. Optimization was in terms of minimizing different variations on the model prediction error as well as some detection performance metrics and the informativeness of the sensors with respect to the image (mutual information). Computationally expedient means of performing the different optimizations were also found. Sharif \& Kamalabadi (2008) demonstrated that rather different results could be obtained depending on the criteria for which the array was optimized.

There are generally good reasons, however, for confining all of the sensors to a plane if possible. If the antennas are above imperfect earth, then their vertical phase centers may not be well known, but at least they will be identical if the antennas are at the same height and the dielectric constant of the earth is homogeneous. Moreover, with $d_{z}=0$ for all interferometry baselines and with the radical in the denominator incorporated into the effective brightness, (2) becomes a two-dimensional Fourier transform. Translations in the brightness consequently map to phase shifts in the visibilities which further map to linear phase progressions of received signals across the aperture plane. This simple relationship can be useful in establishing the absolute phases of signals from different sensors.

\subsection{Phase calibration}

Sensor phase biases associated with differential cable lengths and other systemic issues must be removed through calibration in order for visibilities to be estimated and inverted. While a number of calibration methods exist, calibration remains one of the most challenging aspects of aperture synthesis imaging in practice (see for example Chau et al. (2008)). The "gold standard" for calibration involves observing point targets with known bearings, i.e. radio stars, and adjusting the complex gains of the receiver channels until the measured visibilities match a model based on the known source locations. However, this is only possible for large-aperture radars with adequate sensitivity, and the infrequency of radio star conjunctions may pose practical problems. Feeding common signals through the entire signal chain is another effective calibration strategy, but this too may be possible only infrequently.

Specular meteor echoes are quasi point-targets that can be observed frequently, even by small radars. While their bearings are not known individually, they can be estimated collectively. If the antenna array lies in a plane, phase biases can be estimated such that the bearings of all meteor echoes are consistent across all interferometry baselines (e.g. Holdsworth et al. (2004)). Corrections to the phase bias estimated can then be made such that the center of gravity of the echoes is aligned with the effective two-way radar radiation pattern, with allowances made for the expected anticipated altitude distribution of the specular meteor echoes. Other radar targets may be used to fine-tune the calibration. For example, that echoes from plasma irregularities arise from the locus of magnetic perpendicularity affords accurate knowledge of their elevation along a given azimuth.

While not widely used in upper atmospheric research, closure phase measurements offer additional information for phase calibration (Cornwall, 1989; Jennison, 1958). The idea is that the sum of the visibility phases from a triad of sensors, calculated for instance using bispectral 
(triple-product) analysis, is bias free. If the baseline of the triad are suitably arranged, this can afford some information about sensor phase bias.

A promising class of calibration techniques involves finding the phase biases on the basis of image characteristics through optimization. Uncompensated sensor phase biases tend to degrade images and introduce artifacts that increase the image variance (decrease the smoothness) as well as the overall image entropy. Holding the phase biases of three non-collinear sensors to be arbitrary, the remaining phases could be adjusted to minimize the image entropy, global variance, or some other cost function. Afterward, the phase offsets could be readjusted to "rotate" the artifact-free image into its proper place, taking into account known characteristics of the radar and the target. This optimization can take place outside of the main imaging computation or possibly within it, adopting some of the principles followed by Sharif \& Kamalabadi (2008) for optimizing sensor placement. Their smoothness and support metrics, respectively, could be imposed to accomplish the first and second steps of the aforementioned calibration, respectively, only within a unified imaging framework. Since the brightness/visibility mapping is not linear in the phase biases, the procedure would necessarily be iterative.

\section{Examples from the upper atmosphere}

Here, we present examples of ionospheric phenomena that have been revealed or clarified using aperture synthesis radar imaging. The examples are taken from observations of the Jicamarca Radio Observatory, a 50-MHz phased array radar operated outside Lima, Peru. Aperture synthesis radar imaging was introduced to upper atmospheric research at Jicamarca in 1991 (Kudeki \& Sürücü, 1991), and MaxEnt was applied there first five years later (Hysell, 1996). The number of sensors sampled has grown from four to eight in the intervening years. Twelve-sensor experiments are being planned. The longest interferometry baseline available is nearly 100 wavelengths long in the direction perpendicular to the geomagnetic field. A subset of the main antenna array is used for transmission, and a phase taper is applied to broaden the main beam and reduce the sidelobe level. Images are normally computed over a $\approx 13^{\circ}$-wide azimuth sector. In practice, only the central part of the sector contains echoes and need be plotted.

At Jicamarca, imaging has mainly been applied to coherent scatter from field-aligned plasma density irregularities. Different varieties of irregularities occupy altitudes between about 95-2500 $\mathrm{km}$ at the geomagnetic equator and can be detected by the strong, spectrally narrow radar echoes that arise from them. While imaging is generally performed in two dimensions, the echoes arrive from bearings very close to the locus of perpendicularity to the geomagnetic field, and the images in each range gate can consequently be collapsed into a single dimension. Alternatively, the imaging problem can be formulated in one dimension from the start. Two dimensional images in range and azimuth are produced finally. Sequences of sequential images can also be animated. Imaging in three dimensions has been applied in lower atmospheric applications (Palmer et al., 1998). We have plans to apply it to mesospheric echoes as well.

Images are formed for each Doppler bin, and each image pixel or voxel consequently represents a complete Doppler spectrum. Spectral information is conveyed through color according to the example legend shown in Figure 1. Pixel colors represent the first three moments of the spectrum, with the brightness, hue, and saturation specifying the 


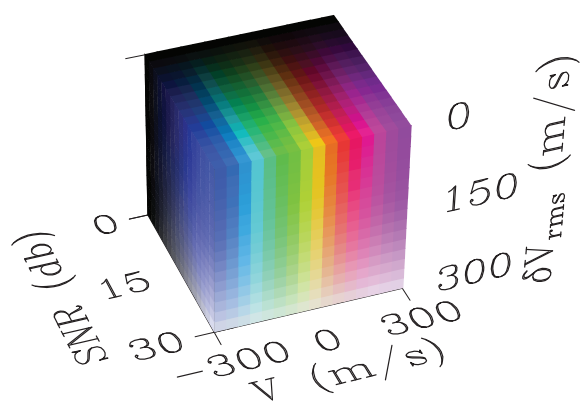

Fig. 1. Scale for interpreting the image pixel coloration. Pixel brightness, hue, and saturation are proportional to the echo signal-to-noise ratio in $\mathrm{dB}$, Doppler shift in $\mathrm{m} / \mathrm{s}$, and spectral width in $\mathrm{m} / \mathrm{s}$, respectively. Different axes ranges apply to the different images that follow.

signal-to-noise ratio, Doppler shift, and spectral width, respectively. In this example, signal-to-noise ratios between $0-30 \mathrm{~dB}$ are represented. The range of Doppler velocities evaluated is controlled by the radar interpulse period and is $300 \mathrm{~m} / \mathrm{s}$ in this example. By convention, RMS spectral widths between zero and the maximum Doppler shift are portrayed. Incoherent integration times for imaging are typically on the order of a few seconds.
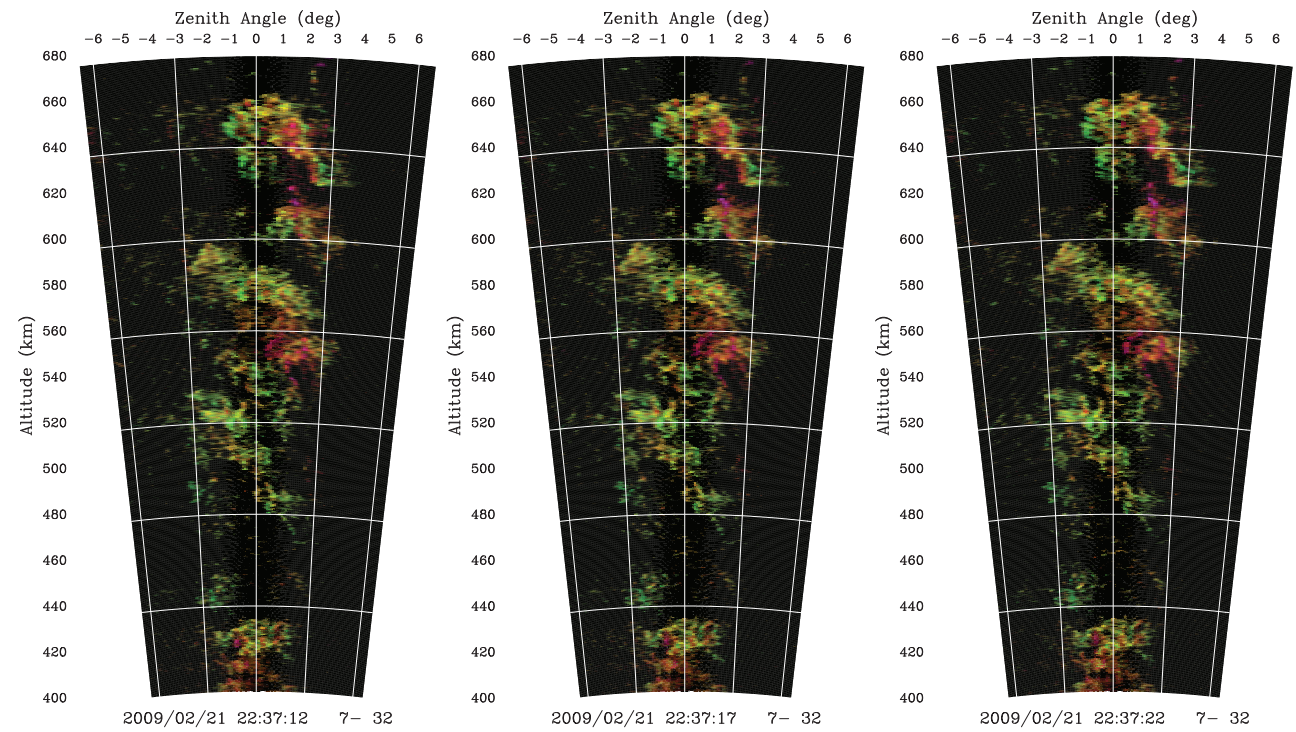

Fig. 2. Coherent scatter from $F$ region plasma density irregularities associated with equatorial spread $F$ depletions on Feb. 21, 2009. The maximum unaliased Doppler velocity is $300 \mathrm{~m} / \mathrm{s}$. Signal-to-noise ratios shown span 7-32 dB.

Figure 2 shows radar images of field-aligned plasma density irregularities associated with equatorial spread $F$ (ESF), a nighttime phenomenon characterized by plasma interchange instability and the rapid ascent of depleted plasma wedges from the $F$ region bottomside 
into the topside. The depletions appear as tilted plumes in the equatorial plane. Here, five plumes between $480-660 \mathrm{~km}$ altitude and separated horizontally by about $40 \mathrm{~km}$ subtend the Jicamarca beam. No other instrument can provide two-dimensional imagery of ESF plumes with details in the crucial intermediate-scale regime (kilometers to tens of kilometers). Animated sequences of images provide dynamical information with the same detail.

Conventional range-time-intensity (RTI) representations of coherent scatter from ESF demonstrate tremendous qualitative variability from event to event, whereas different plumes generally appear to be similar in radar imagery. RTI plots have been likened to slit-camera images from a "photo finish," which may produce spurious evidence of horses with three or five legs from time to time, for example (Woodman, 1997). Aperture synthesis imaging reduces instrumental distortion, revealing the salient features of the phenomena under study.

Comparisons with in situ observations of ESF have shown that the bright patches in Figure 2 correspond to localized plasma depletions (Hysell et al., 2009). Moreover, the Doppler shifts of those patches correspond closely to the vertical components of the local $\mathbf{E} \times \mathbf{B}$ drifts. However, since the coherent scatter is spatially intermittent and not homogeneous, the Doppler spectrum representing an entire range gate (without imaging) will not be indicative of the average line-of-sight speed of the plasma in that gate. We know this intuitively; even though the Doppler shifts from active ESF predominantly denote ascent, by mass and by volume, the action of interchange instability is to push ionospheric plasma downward.
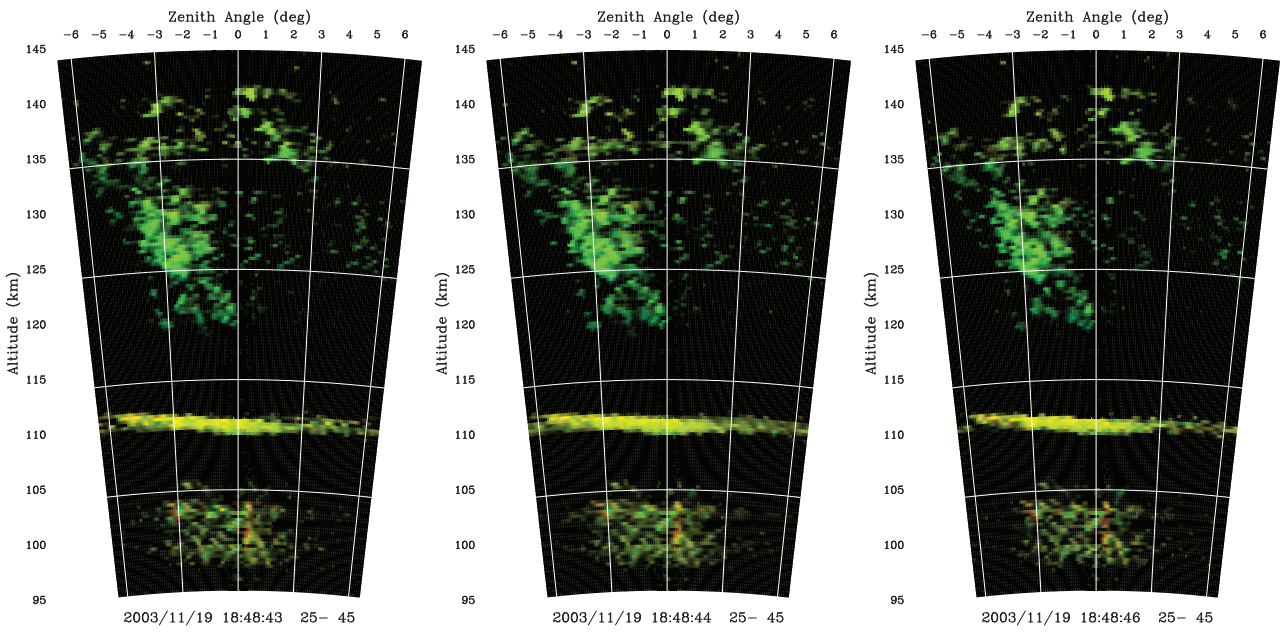

Fig. 3. Coherent scatter from the $E$ and valley regions near twilight on Nov. 19, 2003. The maximum unaliased Doppler velocity is $120 \mathrm{~m} / \mathrm{s}$. Signal-to-noise ratios shown span 25-45 dB.

Figure 3 shows coherent scatter imagery from around twilight when strong echoes were observed between about 120-145 km in the equatorial valley region (Chau \& Hysell, 2004). The echoes do not appear to be directly connected with the equatorial electrojet, which is also producing irregularities below about $112 \mathrm{~km}$ here. The valley echoes are organized into waves with wavelengths of about $10 \mathrm{~km}$ which propagate downward and westward. The Doppler shifts are mainly positive (downward) and vary systematically with height. It would have 
been difficult to distinguish this phenomenon from electrojet-related plasma waves on the basis of RTI information alone.

The cause of the echoes and the underlying source of free energy have not been identified. Recent simulations suggest that the marginal magnetization of the ions at these altitudes is significant and that the irregularities may be due to a class of collisional drift waves. Radar imaging will play a key role in the ongoing investigation of these irregularities.
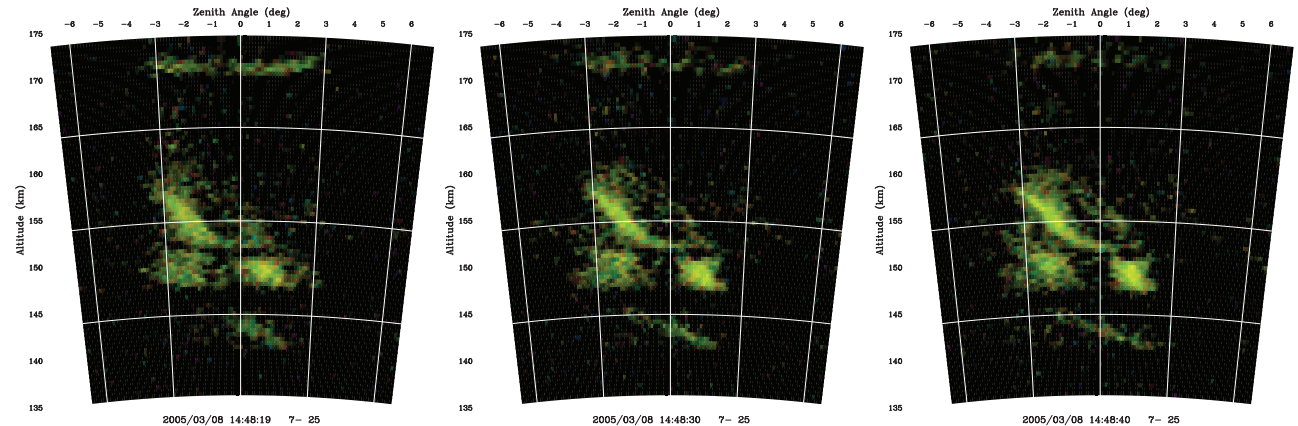

Fig. 4. Radar images of so-called "150-km echoes" observed in the afternoon of Mar. 8, 2005. The maximum unaliased Doppler velocity is $780 \mathrm{~m} / \mathrm{s}$. Signal-to-noise ratios shown span 7-25 dB.

Figure 4 presents radar images associated with the so-called "150-km" echoes (Chau, 2004; Kudeki \& Fawcett, 1993; Royrvik \& Miller, 1981). These daytime echoes are enigmatic, having regular and striking but unexplained patterns in RTI representations. The Doppler shifts of the echoes are known to match the background line-of-sight $\mathbf{E} \times \mathbf{B}$ drift, implying that dielectric plasma polarization probably does not play a significant role in irregularity production. The spectra, in fact, conform in many ways to expectation for incoherent scatter, both looking perpendicular to $B$ and obliquely to $B$, and part of the $150-\mathrm{km}$ echoes constitute an ion-line enhancement Chau et al. (2009). The most obvious source of free energy for the irregularities is photoelectron production, which peaks nearby, but the mechanisms at work have yet to be articulated.

The 150-km echoes are weak compared to echoes from other equatorial plasma density irregularities. The image in Figure 4 reveals that the echoes are not homogeneous or beam filling but are instead spatially (and temporally) intermittent. Over time, the spatial organization of the echoes in the imagery varies abruptly in a way that does not convey the sense of proper motion.

Lastly, Figure 5 shows images of large-scale waves in the daytime equatorial electrojet (Farley, 1985; Farley \& Balsley, 1973; Kudeki et al., 1982). Coherent scatter from the electrojet is the strongest radar target in the upper atmosphere at VHF frequencies and is produced by a combination of gradient-drift and Farley-Buneman instability. Here, large-scale gradient drift waves with wavelengths of $1-2 \mathrm{~km}$ can be seen propagating westward under the influence of a sheared zonal electron $\mathbf{E} \times \mathbf{B}$ flow associated with a Cowling conductivity. Echoes come from gradient drift wave turbulence and from small-scale, secondary Farley-Buneman waves, with large-telltale Doppler shifts. At night, the flow and the propagation direction reverse, and the wavelength of the dominant large-scale waves increases. 

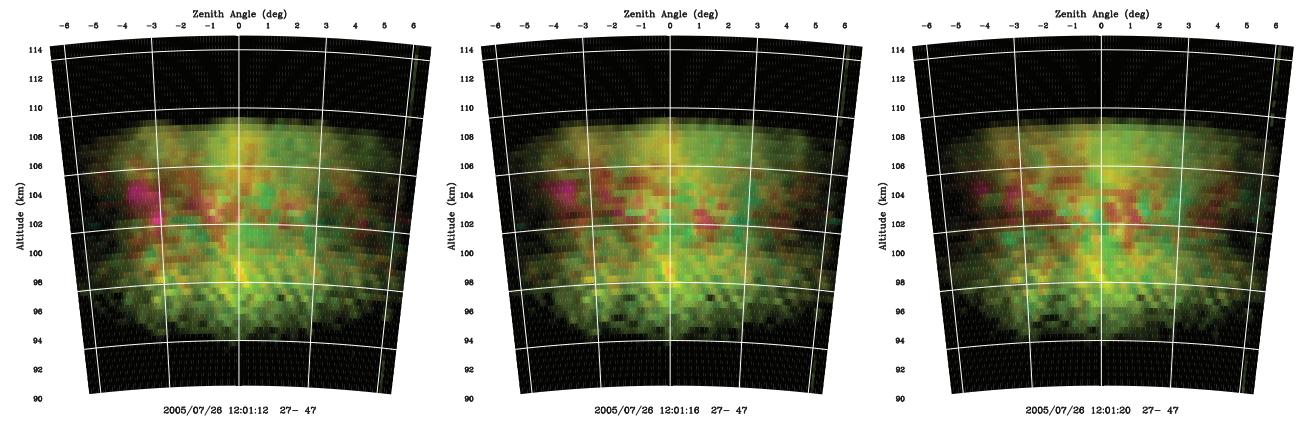

Fig. 5. Coherent scatter from large-scale gradient drift waves in the daytime equatorial electrojet on July 26, 2005. The maximum unaliased Doppler velocity is $600 \mathrm{~m} / \mathrm{s}$.

Signal-to-noise ratios shown span 27-47 dB.

The dominant wavelength of the waves, their phase speeds, and their dynamical behavior are controlled by a surprisingly complex combination of quasilinear, nonlocal, and nonlinear effects which have been described and simulated by Ronchi et al. (1989) and Hu \& Bhattacharjee (1998). Those results could be validated with an unusually high degree of detail using radar imaging experiments (Hysell \& Chau, 2002).

\section{Summary and future work}

Aperture synthesis radar imaging is applied routinely in observations of field-aligned plasma density irregularities at low, middle, and high geomagnetic latitudes, and applications in the lower and middle atmospheres are gradually emerging. Imaging discriminates targets in bearing with resolution limited by the longest interferometry baseline length (rather than by the size of the main antenna) and by the signal-to-noise ratio. It is well suited for heterogeneous sensor arrays with regular or pseudo-random distributions and works alongside other radar modalities like pulse compression and methods for spectral estimation of overspread targets.

We have found the MaxEnt algorithm to be suitable for radar applications in upper atmospheric research. It is an edge-preserving technique, equally applicable to point and continuous targets, and is the embodiment of Occam's razor, suppressing image features for which there is no support in the data. While it was once considered computationally expensive, ongoing improvements in computer performance have made real-time application practical on multi-core systems.

Whereas the example imagery shown above was rendered in two dimensions, imaging is generally performed in three. In the case of scatter from field-aligned plasma density irregularities, information in the direction parallel to the geomegnetic field is limited and is often ignored. However, the magnetic aspect sensitivity of the irregularities, the concentration of the echoes near the locus of perpendicularity, can give insights into the underlying generation mechanism (e.g. Kudeki et al. (1981)). Radar imaging could be used to measure magnetic aspect sensitivity under different conditions in the different regions in the radar field of view. This could be particularly revealing in the auroral electrojet, for example, where different irregularity-producing instabilities may be excited inside, outside, and at the edges of discrete auroral arcs. 
An obvious extension of the algorithms described in this paper involves radar imaging of moderately overspread targets, ESF being a good example. Such targets can be investigated using aperiodic or incremental-lag pulses (e.g. Chau et al. (2004); Uppala \& Sahr (1994); Virtanen et al. (2009)). This produces temporal lagged-products with nonuniform spacing which can be spectrally analyzed using the same methodologies developed for imaging (Hysell et al., 2008). Moreover, the spatio-temporal lagged products arising from aperiodic aperture synthesis imaging experiments can be analyzed together in one operation, yielding images in range, bearing, and the added dimension of Doppler frequency seamlessly. The total number of distinct lagged products will be given by $N(N-1) / 2, N$ being the product of the number of sensors and the number of aperiodic pulses considered at a time. Compromises in spectral and angular resolution will be required if the inverse problem is to remain tractable.

Finally, it has been our observation that the backscatter from ionospheric plasma density irregularities in many contexts tends to be "clumpy" rather than diffuse. This information could be exploited in the inversion scheme if the prior probability function were augmented with a component based on an appropriate model Markov chain. Fully exploiting this information would require processing data from multiple ranges and Doppler frequency bins simultaneously, since the spatial organization occurs equally in bearing, range, and Doppler frequency. That the "clumpy" targets often remain organized for long periods of time suggests that this information should be folded in as well.

\section{References}

Ables, J. G. (1974). Maximum entropy spectral analysis, Astron. Astrophys. Suppl. Ser. 15: 383.

Aster, R. C., Borchers, B. \& Thurber, C. H. (2005). Parameter Estimation and Inverse Problems, Elsevier, New York.

Capon, J. (1969). High-resolution frequency-wavenumber spectrum analysis, Proc. IEEE 57: 1408.

Chau, J. L. (2004). Unexpected spectral characteristics of VHF radar signals from 150-km region over Jicamarca, Geophys. Res. Lett. 31, L23803: doi:10.1029/2004GL021620.

Chau, J. L. \& Hysell, D. L. (2004). High altitude large-scale plasma waves in the equatorial electrojet at twilight, Ann. Geophys. 22: 4071.

Chau, J. L., Hysell, D. L., Kuyeng, K. M. \& Galindo, F. (2008). Phase calibration approaches for radar interferometry and imaging configurations: Equatorial spread $F$ results, Ann. Geophys. 26: 2333-2343.

Chau, J. L., Hysell, D. L., Reyes, P. M. \& Milla, M. A. (2004). Improved spectral estimations of equatorial spread $F$ echoes at Jicamarca using aperiodic transmitter coding, J. Atmos. Sol. Terr. Phys. 66(1543).

Chau, J. L. \& Woodman, R. F. (2001). Three-dimensional coherent radar imaging at Jicamarca: Preliminary results, J. Atmos. Sol. Terr. Phys. 63: 253-261.

Chau, J. L. \& Woodman, R. F. (2004). Observations of meteor-head echoes using the Jicamarca $50 \mathrm{MHz}$ radar in interferometer mode, Atmos. Chem. Phys. 4.1680-7324/acp: 511-521.

Chau, J. L., Woodman, R. F., Milla, M. A. \& Kudeki, E. (2009). Naturally enhanced ion-line spectra around the equatorial 150-km region, Ann. Geophys. 27: 933-942.

Cornwall, T. J. (1989). The applications of closure phase to astronomical imaging, Science 245 (4915): 263-269.

Cornwell, T. J. \& Evans, K. F. (1985). A simple maximum entropy deconvolution algorithm, Astron. Astrophys. 143: 77. 
Daniell, G. J. (1991). Of maps and monkeys, in B. Buck \& V. A. Macaulay (eds), Maximum Entropy in Action, Clarendon, Oxford, chapter 1, pp. 1-18.

Farley, D. T. (1969). Incoherent scatter correlation function measurements, Radio Sci. 4: 935-953.

Farley, D. T. (1972). Multiple-pulse incoherent-scatter correlation function measurements, Radio Sci. 7: 661.

Farley, D. T. (1985). Theory of equatorial electrojet plasma waves: New developments and current status, J. Atmos. Terr. Phys. 47: 729-744.

Farley, D. T. \& Balsley, B. B. (1973). Instabilities in the equatorial electrojet, J. Geophys. Res. 78: 227.

Farley, D. T. \& Hysell, D. L. (1996). Radar measurements of very small aspect angles in the equatorial ionosphere, J. Geophys. Res. 101: 5177.

Gull, S. F. \& Daniell, G. J. (1978). Image reconstruction from incomplete and noisy data, Nature 272: 686 .

Hassenpflug, G., Yamamoto, M., Luce, H. \& Fukao, S. (2008). Description and demonstration of the new Middle and Upper atmosphere Radar imaging system: 1-D, 2-D, and 3-D imaging of troposphere and stratosphere " Radio Sci. 43, RS2013: doi:10.1029/2006RS003603.

Högbom, J. A. (1974). Aperture synthesis with a non-regular distribution of interferometer baselines, Astron. Astrophys. Supp. 15: 417-426.

Holdsworth, D. A., Tsutsumi, M., Reid, I. M., Nakamura, T., T. \& Tsuda (2004). Interferometric meteor radar phase calibration, Radio Sci. 39, RS5012: doi:10.1029/2003RS003026.

$\mathrm{Hu}, \mathrm{S}$. \& Bhattacharjee, A. (1998). Two-dimensional simulations of gradient-drift turbulence in the daytime equatorial electrojet, J. Geophys. Res. 103: 20,749.

Huuskonen, A., Lehtinen, M. S. \& Pirttilä, J. (1996). Fractional lags in alternating codes: Improving incoherent scatter measurements by using lag estimates at noninteger multiples of baud length, Radio Sci. 31: 245.

Hysell, D. L. (1996). Radar imaging of equatorial $F$ region irregularities with maximum entropy interferometry, Radio Sci. 31: 1567.

Hysell, D. L. (2007). Inverting ionospheric radio occultation measurements using maximum entropy, Radio Sci. 42, RS4022: doi:10.1029/2007RS003635.

Hysell, D. L. \& Chau, J. L. (2002). Imaging radar observations and nonlocal theory of large-scale waves in the equatorial electrojet, Ann. Geophys. 20: 1167.

Hysell, D. L. \& Chau, J. L. (2006). Optimal aperture synthesis radar imaging, Radio Sci. 41: 10.1029/2005RS003383, RS2003.

Hysell, D. L., Chau, J. L. \& Lakshmanan, S. (2008). Improved spectral estimation of equatorial spread $F$ through aperiodic pulsing and Bayesian inversion, Radio Sci. 43, RS2010: doi:10.1029/2007RS003790.

Hysell, D. L., Hedden, R. B., Chau, J. L., Galindo, F. R., Roddy, P. A. \& Pfaff, R. F. (2009). Comparing $F$ region ionospheric irregularity observations from C/NOFS and Jicamarca, Geophys. Res. Lett. 36, L00C01: doi:10.1029/2009GL038983.

Jaynes, E. T. (1982). On the rationale of maximum-entropy methods, Proc. IEEE 70: 939.

Jaynes, E. T. (1985). Where do we go from here?, in C. R. Smith \& W. T. Grandy, Jr. (eds), Maximum-Entropy and Bayesian Methods in Inverse Problems, D. Reidel, Norwell, Mass., chapter 2, pp. 21-58. 
Jennison, R. C. (1958). A phase sensitive interferometer technique for the measurement of the Fourier transforms of spatial brightness distributions of small angular extent, Mon. Not. Roy. Astron. Soc. 118: 276.

Kosarev, E. L. (1990). Shannon's superresolution limit for signal recovery, Inverse Problems 6: 55-76.

Kudeki, E., Farley, D. T. \& Fejer, B. G. (1982). Long wavelength irregularities in the equatorial electrojet, Geophys. Res. Lett. 9: 684.

Kudeki, E. \& Fawcett, C. D. (1993). High resolution observations of $150 \mathrm{~km}$ echoes at Jicamarca, Geophys. Res. Lett. 20: 1987.

Kudeki, E., Fejer, B. G., Farley, D. T. \& Ierkic, H. M. (1981). Interferometer studies of equatorial F region irregularities and drifts, Geophys. Res. Lett. 8: 377.

Kudeki, E. \& Sürücü, F. (1991). Radar interferometric imaging of field-aligned plasma irregularities in the equatorial electrojet, Geophys. Res. Lett. 18: 41.

Kudeki, E. \& Woodman, R. F. (1990). A post-statistics steering technique for MST radar applications, Radio Sci. 25: 591-594.

Lehtinen, M. S. (1986). Statistical theory of incoherent scatter radar measurements, Technical Report 86/45, Eur. Incoherent Scatter Sci. Assoc., Kiruna, Sweden.

Lomb, N. R. (1976). Least squares frequency analysis of unevenly sampled data, Astrophys. Space Sci. 39: 447.

Menke, W. (1984). Geophysical Data Analysis: Discrete Inverse Theory, Academic, New York.

Palmer, R. D., Gopalam, S., Yu, T. Y. \& Fukao, S. (1998). Coherent radar imaging using Capon's method, Radio Sci. 33: 1585.

Powell, M. J. D. (1970). A hybrid method for nonlinear equations, in P. Rabinowitz (ed.), Numerical Methods for Nonlinear Algebraic Equations, Gordon and Breach, London, pp. 87-114.

Ronchi, C., Similon, P. L. \& Sudan, R. N. (1989). A nonlocal linear theory of the gradient drift instability in the equatorial electrojet, J. Geophys. Res. 94: 1317.

Royrvik, O. \& Miller, K. L. (1981). Nonthermal scattering of radio waves near $150 \mathrm{~km}$ above Jicamarca, Peru, J. Geophys. Res. 86: 180.

Schmidt, R. O. (1986). Multiple emitter location and signal parameter estimation, IEEE Trans. Antennas Propagat. AP-34: 276-280, March.

Semeter, J., Butler, T., Heinselman, C., Nicolls, M., Kelly, J. \& Hampton, D. (2009). Volumetric imaging of the auroral ionosphere: Initial results from PFISR, J. Atmos. Sol. Terr. Phys. 71(6-7): 738-743.

Shannon, C. E. \& Weaver, W. (1949). The Mathematical Theory of Communication, Univ. of Ill. Press, Urbana.

Sharif, B. \& Kamalabadi, F. (2008). Optimal sensor array configuration in remote image formation, IEEE Trans. Image Proc. 17(2): 155-166.

Silver, R. N., Sivia, D. S. \& Gubernatis, J. E. (1990). Maximum-entropy method for analytic continuation of quantum Monte Carlo data, Phys. Rev. 41(4): 2380.

Skilling, J. (1989). Classic maximum entropy, in J. Skilling (ed.), Maximum Entropy and Bayesian Methods, Kluwer Academic Publishers, Dordtrecht, pp. 45-52.

Skilling, J. (1991). Fundamentals of MaxEnt in data analysis, in B. Buck \& V. A. Macaulay (eds), Maximum Entropy in Action, Clarendon, Oxford, chapter 2, pp. 19-40.

Skilling, J. \& Bryan, R. K. (1984). Maximum entropy image reconstruction: General algorithm, Mon. Not. R. Astron. Soc. 211: 111. 
Sulzer, M. P. (1986). A radar technique for high range resolution incoherent scatter autocorrelation function measurements utilizing the full average power of klystron radars, Radio Sci. 21: 1033-1040.

Tarantola, A. (1987). Inverse Theory, Elsevier, New York.

Thompson, A. R. (1986). Interferometry and Synthesis in Radio Astronomy, John Wiley, New York.

Uppala, S. V. (1993). Aperiodic radar technique for the spectrum estimation of moderately overspread targets, Master's thesis, University of Washington.

Uppala, S. V. \& Sahr, J. D. (1994). Spectrum estimation moderately overspread radar targets using aperiodic transmitter coding, Radio Sci. 29: 611.

Virtanen, I. I., Viernen, J. \& Lehtinen, M. S. (2009). Phase-coded pulse aperiodic transmitter coding, Ann. Geophys. 27: 2799-2811.

Wernecke, S. J. \& D'Addario, L. R. (1977). Maximum entropy image reconstruction, IEEE Trans. Computers c-26: 351.

Wilczek, R. \& Drapatz, S. (1985). A high accuracy algorithm for maximum entropy image restoration in the case of small data sets, Astron. Astrophys. 142: 9.

Woodman, R. F. (1997). Coherent radar imaging: Signal processing and statistical properties, Radio Sci. 32: 2373.

Woodman, R. F. (2009). Spread F- An old equatorial aeronomy problem finally resolved?, Ann. Geophys. 27: 1915-1934.

Woodman, R. F. \& La Hoz, C. (1976). Radar observations of $F$ region equatorial irregularities, J. Geophys. Res. 81: 5447-5466.

Wu, N. (1984). A revised Gull-Daniell algorithm in the maximum entropy method, Astron. Astrophys. 139: 555. 


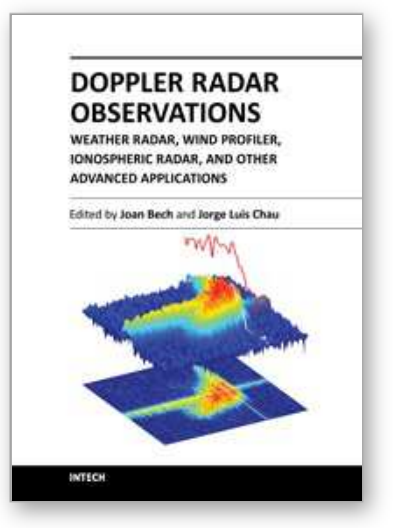

\author{
Doppler Radar Observations - Weather Radar, Wind Profiler, \\ lonospheric Radar, and Other Advanced Applications \\ Edited by Dr. Joan Bech
}

ISBN 978-953-51-0496-4

Hard cover, 470 pages

Publisher InTech

Published online 05, April, 2012

Published in print edition April, 2012

Doppler radar systems have been instrumental to improve our understanding and monitoring capabilities of phenomena taking place in the low, middle, and upper atmosphere. Weather radars, wind profilers, and incoherent and coherent scatter radars implementing Doppler techniques are now used routinely both in research and operational applications by scientists and practitioners. This book brings together a collection of eighteen essays by international leading authors devoted to different applications of ground based Doppler radars. Topics covered include, among others, severe weather surveillance, precipitation estimation and nowcasting, wind and turbulence retrievals, ionospheric radar and volcanological applications of Doppler radar. The book is ideally suited for graduate students looking for an introduction to the field or professionals intending to refresh or update their knowledge on Doppler radar applications.

\title{
How to reference
}

In order to correctly reference this scholarly work, feel free to copy and paste the following:

D.L. Hysell and J.L. Chau (2012). Aperture Synthesis Radar Imaging for Upper Atmospheric Research, Doppler Radar Observations - Weather Radar, Wind Profiler, lonospheric Radar, and Other Advanced Applications, Dr. Joan Bech (Ed.), ISBN: 978-953-51-0496-4, InTech, Available from:

http://www.intechopen.com/books/doppler-radar-observations-weather-radar-wind-profiler-ionospheric-radarand-other-advanced-applications/aperture-synthesis-radar-imaging

\section{INTECH}

open science | open minds

\author{
InTech Europe \\ University Campus STeP Ri \\ Slavka Krautzeka 83/A \\ 51000 Rijeka, Croatia \\ Phone: +385 (51) 770447 \\ Fax: +385 (51) 686166 \\ www.intechopen.com
}

\author{
InTech China \\ Unit 405, Office Block, Hotel Equatorial Shanghai \\ No.65, Yan An Road (West), Shanghai, 200040, China \\ 中国上海市延安西路65号上海国际贵都大饭店办公楼405单元 \\ Phone: +86-21-62489820 \\ Fax: +86-21-62489821
}


(C) 2012 The Author(s). Licensee IntechOpen. This is an open access article distributed under the terms of the Creative Commons Attribution 3.0 License, which permits unrestricted use, distribution, and reproduction in any medium, provided the original work is properly cited. 